\title{
Isothermal Langevin dynamics in systems with power-law spatially-dependent friction
}

\author{
Shaked Regev, ${ }^{1}$ Niels Grønbech-Jensen, ${ }^{2,3}$ and Oded Farago ${ }^{1,4}$ \\ ${ }^{1}$ Department of Biomedical Engineering, Ben-Gurion University of the Negev, Be'er Sheva 85105, Israel \\ ${ }^{2}$ Department of Mechanical and Aerospace Engineering, \\ University of California, Davis, California 95616, USA \\ ${ }^{3}$ Department of Mathematics, University of California, Davis, California 95616, USA \\ ${ }^{4}$ Ilse Katz Institute for Nanoscale Science and Technology, \\ Ben-Gurion University of the Negev, Be'er Sheva 85105, Israel
}

\begin{abstract}
We study the dynamics of Brownian particles in a heterogeneous one-dimensional medium with a spatially-dependent diffusion coefficient of the form $D(x) \sim|x|^{c}$, at constant temperature. The particle's probability distribution function $(\mathrm{PDF})$ is calculated both analytically, by solving Fick's diffusion equation, and from numerical simulations of the underdamped Langevin equation. At large times, the PDFs calculated by both approaches yield identical results, corresponding to subdiffusion for $c<0$, and superdiffusion for $0<c<1$. For $c>1$, the diffusion equation predicts that the particles accelerate. Here, we show that this phenomenon, previously considered in several works as an illustration for the possible dramatic effects of spatially-dependent thermal noise, is unphysical. We argue that in an isothermal medium, the motion cannot exceed the ballistic limit $\left(\left\langle x^{2}\right\rangle \sim t^{2}\right)$. The ballistic limit is reached when the friction coefficient drops sufficiently fast at large distances from the origin, and is correctly captured by Langevin's equation.
\end{abstract}

\section{INTRODUCTION}

Brownian motion was first observed by the botanist Robert Brown while examining, under a microscope, the motion of pollen grains and noticing their random jitter in water [1]. Years later, an explanation for this observation was given by Albert Einstein, who traced it to the random collisions between the grains and the water molecules [2]. These collisions cause the Brownian particle to exhibit a "random walk" in space - a phenomenon also known as single particle diffusion. Brownian motion can be described by the diffusion equation for the particle's probability density function (PDF), which in one-dimension (1D) reads

$$
\frac{\partial P(x, t)}{\partial t}=D \frac{\partial^{2} P(x, t)}{\partial x^{2}}
$$

where $x$ and $t$ denote, respectively, coordinate and time, and $D$ is the diffusion coefficient. Assuming a Dirac delta-function initial condition $P(x, 0)=\delta(x)$, the solution of Eq. (11) is given by the normal distribution $P(x, t)=(4 \pi D t)^{-0.5} \exp \left(-x^{2} / 4 D t\right)$. The mean displacement of the Brownian particle $\langle\Delta x\rangle=0$, while the mean squared displacement (MSD) grows linearly with time: $\left\langle\Delta x^{2}\right\rangle=2 D t$.

An alternative route for describing the motion of a Brownian particle is the Langevin equation [3]

$$
m \frac{d v}{d t}=-\alpha v+\beta(t)
$$

where $m$ and $v$ denote, respectively, the mass and velocity of the particle. In this description, the impact of the random collisions on the Brownian particle is realized through the action of two forces, represented by the terms on the r.h.s. of the equation. The first is a friction force representing the statistical average of the collision forces, while the second is a random Gaussian white noise accounting for the force distribution around the mean value. Since the magnitude of the collision forces depends on the characteristic thermal velocity of the molecules of the embedding fluid, the friction coefficient $\alpha$ in Langevin's equation (2) must depend on the temperature, $T$. It should also be related to the diffusion coefficient, $D$, appearing in Eq. (1). The relation, $\alpha=k_{B} T / D$ (where $k_{B}$ is Boltzmann's constant), is known as Einstein's relation, which is closely related to the more general fluctuation-dissipation theorem [4]. In order for the latter to be satisfied, one must also assume that the Gaussian white noise term in Langevin's equation has the following statistical properties [5]: $\langle\beta(t)\rangle=0$, and $\left\langle\beta(t) \beta\left(t^{\prime}\right)\right\rangle=2 \alpha k_{B} T \delta\left(t-t^{\prime}\right)$, where $\langle\cdots\rangle$ denotes average over all possible realizations of the noise force $\beta(t)$.

Two comments regarding Langevin's equation (2) should be made. First, the equation describes Brownian diffusive dynamics only at large time scales. On short time scales, Langevin dynamics is ballistic (inertial). The crossover between the ballistic and diffusive regimes occurs at $\tau \sim m / \alpha$. Second, the equation neglects the influence of the motion of the Brownian particle on the embedding fluid. The fluid acts as an ideal heat bath whose properties remain unaffected by the presence of the moving Brownian particle. This latter assumption is justified when the number of fluid molecules is macroscopically large, and when the momentum and energy are locally transferred to the bulk fluid much faster than any other relevant time scale of the dynamics.

The difference between the diffusion equation approach to Brownian dynamics and the Langevin equation formalism becomes more significant when one deals with diffusion in a medium with a position-dependent friction coefficient $\alpha(x)$. These types of dynamics are often associated with the Itô-Stratonovich dilemma [6]. In this paper we study specific examples of such dynamics occuring in systems with power-law diffusion coefficients 
$D(x) \sim|x|^{c}$. Model systems with spatially-dependent diffusivity have been receiving renewed interest recently due to their relevance to single particle experiments involving femto-Newton force measurements [7, 8]. The dilemma itself is not the main topic of this paper, and we refer the reader to textbooks on stochastic dynamics, e.g. 9, 10], for more details. Here we summarize only the highlights relevant to this work:

1. The generalization of Eq. (11) corresponding to dynamics of Brownian particles, at constant temperature, in (1D) systems with spatially-dependent friction coefficients, is [1]

$$
\frac{\partial P(x, t)}{\partial t}=\frac{\partial}{\partial x}\left(D(x) \frac{\partial P(x, t)}{\partial x}\right) .
$$

This is Fick's second law $\partial_{t} P=-\partial_{x} J$, with the flux $J(x, t)=-D(x) \partial_{x} P(x, t)$.

2. The corresponding Langevin equation reads [12]

$$
m \frac{d v}{d t}=-\alpha(x) v+\beta(x(t)),
$$

with $\alpha(x)=k_{B} T / D(x)$, which is a natural generalization of Einstein's relation [13].

3. A dilemma arises when the Langevin equation (4) is integrated over time in order to calculate the trajectory of the particle 14]. Since the particle moves during the infinitesimal time step $d t$, the value of $\alpha(x)$ also changes, and the Langevin equation of motion must be supplemented with a convention (rule) for choosing the value of $\alpha(x)$. The name "Itô-Stratonovich dilemma" assigned to the ambiguity about the choice of interpretation is after the two most commonly used conventions - the one of Itô which uses the value of $\alpha$ at the beginning of the time step, and the one of Stratonovich which takes the average of the friction function at the initial and the end points.

4. In the overdamped limit, i.e., when the inertial term on the l.h.s. of Eq. (4) is set identically to zero, different conventions lead to trajectories with different statistical properties, even for $d t \rightarrow 0$ [15]. For Brownian dynamics at constant temperature, the correct convention that generates the PDF solving Eq. (3) is neither Itô's nor that of Stratonovich, but rather Hänggi's interpretation (also known as the "isothermal" convention) [16, 17] which uses $\alpha$ at the end of the time step [1, 12].

5. In the case of underdamped Langevin dynamics [i.e., Eq. (4) with the l.h.s. not assumed to be vanishingly small], all (reasonable) conventions converge to the correct PDF in the limit $d t \rightarrow 0$. This difference between underdamped dynamics and its overdamped limiting case (see item 4 above) stems from fact that in the latter, the velocity is physically ill-defined (since it is proportional to the white noise $\beta$ ), while in the former, it remains finite and follows the equilibrium Maxwell-Boltzmann distribution. Formally (mathematically) speaking, there is no dilemma in the second-order (in $x$ ) equation (44). However, the rate of convergence of numerical simulationresults toward the theoretical PDF [i.e., the solution of
Eq. (3)] greatly depends on the chosen convention and the numerical integrator. This issue has considerable practical importance in numerical simulations where the time step $d t$ is not infinitesimal. The results in the work are based on Langevin dynamics simulations employing the G-JF integrator [18, 19] with a newly proposed "inertial" convention [12, 13] (see details in section [1B). This combination produces excellent results even for relatively large integration time steps.

With the above in mind, we now turn to examine the behavior of a Brownian particle moving, at constant temperature, in a $1 \mathrm{D}$ system with a power-law diffusion function $D(x)=D_{0}|x / l|^{c}$. In the following section we calculate the PDF of the particle by solving the diffusion equation (3), and by numerically integrating the Langevin equation of motion (44). As we will observe, these two approaches do not necessarily yield similar results.

\section{HETEROGENEOUS MEDIA WITH POWER LAW FRICTION FUNCTION}

\section{A. Fick's second law}

For $D(x)=D_{0}|x / l|^{c}$, the solution of Eq. (3) is

$$
P(x, t)=\frac{\left[(2-c)^{c} D_{0} t\right]^{1 /(c-2)}}{2 \Gamma\left(\frac{1}{2-c}\right)} \exp \left[\frac{-|x|^{2-c}}{(2-c)^{2}\left(D_{0} t\right)}\right],
$$

where $\Gamma$ is the Gamma function, and for brevity we set $l=1$. This solution satisfies the condition that the particle's motion starts at the origin: $P(x, 0)=\delta(x)$. From the requirement that $P(x, t)$ vanishes for $x \rightarrow \pm \infty$, which is necessary (but not sufficient) to ensure that $\int_{-\infty}^{\infty} P(x, t) d x=1$, we infer that the solution can be physical only for $c<2$. From symmetry considerations, the ensemble average $\langle x\rangle=0$, while the MSD,

$\left\langle x^{2}\right\rangle=\int_{-\infty}^{\infty} x^{2} P(x, t) d x=\frac{\Gamma\left(\frac{3}{2-c}\right)}{\Gamma\left(\frac{1}{2-c}\right)}\left[(2-c)^{2} D_{0} t\right]^{2 /(2-c)}$.

Thus, for $c<0$ we observe subdiffusion, and for $0<c<1$ we find superdiffusion. For $c=0$ we have $\left\langle x^{2}\right\rangle=2 D_{0} t$, i.e., normal diffusion, and for $c=1$ the particle's motion is ballistic. For $c>1$, Eq. (6) predicts dynamics which are faster than ballistic (for instance, $c=1.5$ corresponds to dynamics at costant acceleration). This is an unphysical result, and in what follows we demonstrate that for any $c \geq 1$ the motion remains ballistic.

\section{B. Langevin Dynamics Simulations}

The PDF can be obtained from an ensemble of trajectories of particles starting at the origin, $x^{0}=0$, with 
initial velocities, $v^{0}$, drawn from an equilibrium MaxwellBoltzmann distribution. The trajectories are computed by numerically integrating Langevin's equation of motion (44). Denoting, respectively, by $x^{n}$ and $v^{n}$ the position and velocity of a particle at time $t_{n}$, the integration is conducted using the G-JF algorithm that advances the system by one time step to $t_{n+1}=t_{n}+d t$, using the following set of discrete-time equations [18, 19]:

$$
\begin{aligned}
& x^{n+1}=x^{n}+b d t v^{n}+\frac{b d t^{2}}{2 m} f^{n}+\frac{b d t}{2 m} \beta^{n+1} \\
& v^{n+1}=a v^{n}+\frac{d t}{2 m}\left(a f^{n}+f^{n+1}\right)+\frac{b}{m} \beta^{n+1},
\end{aligned}
$$

where $f^{n}=f\left(x^{n}\right)$ is the deterministic force acting on the particle, $\beta^{n+1}$ is a Gaussian random number with

$$
\left\langle\beta^{n}\right\rangle=0 ;\left\langle\beta^{n} \beta^{l}\right\rangle=2 \alpha k_{B} T d t \delta_{n, l},
$$

and the damping coefficients of the algorithm are

$$
b=[1+(\alpha d t / 2 m)]^{-1} ; a=[1-(\alpha d t / 2 m)] b .
$$

We set $f^{n}=0$ since we consider the case when the particle experiences no forces other than random collisions with the fluid molecules.

Since the friction coefficient varies in space, the above equations (7) and (8) must be complemented with a convention for choosing the value of $\alpha$ to be used in Eqs.(9) and (10) at each time step. Here, we use the recently proposed inertial convention that assigns to $\alpha$ the value of the spatial average of the friction function along the inertial trajectory from $x^{n}$ to $\tilde{x}^{n+1}=x^{n}+v^{n} d t$ [12, 13]

$$
\frac{\int_{x^{n}}^{\tilde{x}^{n+1}} \alpha(x) d x}{\tilde{x}^{n+1}-x^{n}}=\frac{A\left(\tilde{x}^{n+1}\right)-A\left(x^{n}\right)}{\tilde{x}^{n+1}-x^{n}},
$$

where $A(x)$ is the primitive function of $\alpha(x)$. We have previously demonstrated that the combination of the GJF algorithm with the inertial convention produces excellent agreement between the computed and theoretical PDFs, even for relatively large intergration time steps.

Fig. 1 depicts our results for the PDF for systems with a power law friction function $\alpha(x)=k_{B} T / D(x)=$ $\left(k_{B} T / D_{0}\right)|x / l|^{-c}$, for $c=-0.5(\mathrm{~A})$ and $c=+0.5(\mathrm{~B})$. For convenience, we set $m=1, D_{0}=1, k_{B} T=1$, and $l=1$. The results have been obtained from simulations of $2.5 \times 10^{5}$ trajectories with integration time step $d t=10^{-3}$. The open circles in Figs. 1(A) and (B) represent our numerical results for the PDF at $t=1000$ for $c=-0.5$ and $c=0.5$, respectively. The numerical results exhibit excellent agreements with the corresponding analytical predictions of Eq. (5), which are plotted with the solid curves.

The symbols in Fig. 1(C) represent the numerical results for the MSD for $c=-0.5,0.25,0.5,0.75$. We observe that the power-law behavior $\left\langle x^{2}\right\rangle \sim t^{\frac{2}{2-c}}$ [see Eq. [6)], which is depicted by the lines in the figure, is indeed recovered at large times. The same powerlaw (60) was previously derived in ref. 20], where instead of Fick's law (3), a different diffusion equation
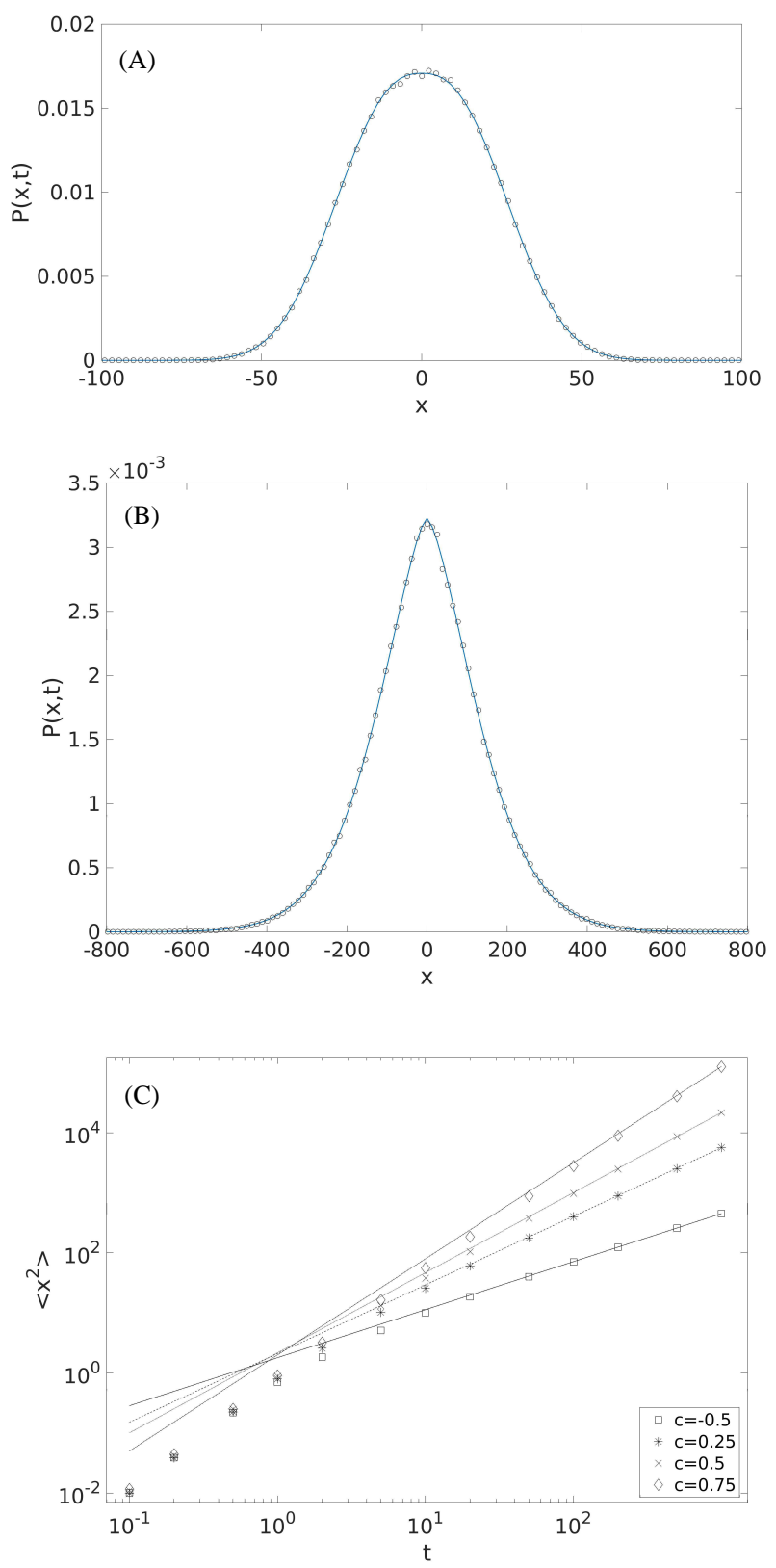

FIG. 1: (A) The PDF at $t=1000$ obtained from the numerical integration (open circles) vs. the PDF given by Eq. (5) (solid line), which solves Fick's diffusion equation for $c=-0.5$. (B) Same as (A), for $c=0.5$. (C) The MSD $\left\langle x^{2}\right\rangle$ of the particle as a function of time for $c=-0.5,0.25,0.5,0.75$ (markers), vs. the expected $\left\langle x^{2}\right\rangle$ for $c=-0.5,0.25,0.5,0.75$ according to Eq. (6) (lines).

$\partial_{t} P=\partial_{x}\left[\sqrt{D(x)} \partial_{x}(\sqrt{D(x)} P)\right]$ was considered. The latter form of the diffusion equation corresponds to the Stratonovich interpretation of the overdamped Langevin equation. The reader is reminded (see section \) that for overdamped Langevin dynamics, different conventions lead to different PDFs. Indeed, although both equations yield the same power-law for the MSD, the PDFs solving these equations look markedly different. Specifically, 
the PDFs of the Statonovich diffusion equation diverge at the origin for $c>0$, and assume a a bimodal form for $c<0$, with a vanishing value at the origin [20]. In contrast, the PDFs of Fick's law of diffusion (which corresponds to Hänggi's interpretation) attain a maximum at the origin. Our Langevin dynamics simulations, which at large times reproduce PDFs that agree with Eq. (5), serve as yet another demonstration for the appropriateness of Hänggi interpretation and Fick's second law for diffusion at constant temperature. This is because the simulations follow the underdamped (inertial) Langevin dynamics of the particle. As noted above, for the inertial Langevin equation, all interpretations converge to the correct solution in the limit of small integration time steps.

\section{BALLISTIC MOTION}

\section{A. The case $c \geq 1$}

Integrating Eq. (4) from the initial time to $t$, and taking the ensemble average over all noise realizations, yields the following relationship

$$
\langle m \Delta v\rangle=-\langle\Delta A(x)\rangle
$$

between the momentum change (force impulse) and displacement of the particle. Eq. (12), which was previously derived in ref. [13], involves $A(x)$ - the primitive function of $\alpha(x)$ [see Eq. (11)]. This implies that $\alpha(x)$ must be an integrable function. For $\alpha(x) \sim x^{-c}$ with $c \geq 1$, the friction function is non-integrable at $x=0$. This feature makes it impossible to start the simulations when the particle is at the origin due to the inability to define the friction coefficient for the initial step. If the particle is placed on one side of the system, it will never cross to the other side. This is because no matter how close the particle approaches to the origin, its ballistic distance (the characteristic distance that it travels before changing its direction) will always be shorter than the distance to the origin. In other words, for $c \geq 1$, the dissipation near the origin diverges so rapidly, that the singularity acts like a wall that stops the particle and bounces it back. This scenario, however, is unphysical, and it stems from the unphysical nature of Langevin's equation which only considers the influence of the medium on the particle but ignores the impact of the particle on the medium. From momentum conservation we know that any change in the momentum of the Brownian particle must be countered by an opposite change in the momentum of the molecules of the medium. This implies that when the particle is reflected from the origin, it exerts a force on the friction singularity, and this force will cause changes in the medium that would not allow the singularity to be long-lived.

Apart from the divergence of $A(x)$ at the origin, it is also interesting to consider the ramifications of the rapid drop in $\alpha(x)$ in the limits $x \rightarrow \pm \infty$. For $c>1$, the integral over $\alpha(x)$ from $x_{0}>0\left(x_{0}<0\right)$ to $+\infty$ $(-\infty)$ is finite, implying that the particle's ballistic distance may diverge. This can be inferred from Eq. (12), which suggests that it is unlikely for a particle to change its direction of motion, if it reaches $x_{0}$ with velocity $v_{0}>\left[A(\infty)-A\left(x_{0}\right)\right] / m$. In other words - as the particle travels further away from the origin, it experiences a vanishingly small friction force and, therefore, its motion would ultimately become ballistic. The crossover from diffusive to ballistic dynamics is further explored in the following section.

\section{B. Crossover to ballistic motion}

We now consider dynamics in a one-dimensional system with the spatially-dependent diffusion coefficient

$$
D(x)=D_{0}\left[1+\left(\frac{x}{l}\right)^{2}\right] .
$$

For this choice, $D(x) \sim x^{c}$ with $c=2$ for $x / l \gg 1$; but unlike the power-law form discussed in section III A above, the friction coefficient, $\alpha(x)=k_{B} T / D(x)$, does not diverge at the origin. A special reason for choosing the specific form Eq. (13) is that it has been given in ref. 11] as an example of a spatially-dependent diffusion coefficient causing increasing acceleration. This result is obtained by multiplying by $x^{2}$ both sides of the diffusion equation (for $D_{0}=1$ and $l=1$ )

$$
\frac{\partial P(x, t)}{\partial t}=\frac{\partial}{\partial x}\left[\left(1+x^{2}\right) \frac{\partial P(x, t)}{\partial x}\right],
$$

and integrating with respect to $x$, which yields the equation

$$
\frac{\partial\left\langle x^{2}\right\rangle}{\partial t}=2+6\left\langle x^{2}\right\rangle
$$

which has the solution

$$
\left\langle x^{2}\right\rangle=[\exp (6 t)-1] / 3 .
$$

However, the prediction of Eq. (16) that the MSD grows exponentially with $t$ is unphysical since it implies the emergence of two opposite currents of particles with everincreasing velocities. One should wonder about the energy source of the exponential growth in the kinetic energy of the Brownian particles. The particles are immersed in a medium of uniform temperature serving as a heat bath, and experience no force other than random collisions with the molecules of the medium. It is impossible that through random collisions, the Brownian particles would consistently gain energy allowing them to reach exponentially large speeds, especially at large distances where the friction coefficient vanishes, which means that the rate of collisions with the heat bath becomes increasingly small.

The erroneous Eq. (16) is derived from the diffusion equation (14). The latter, however, does not correctly 

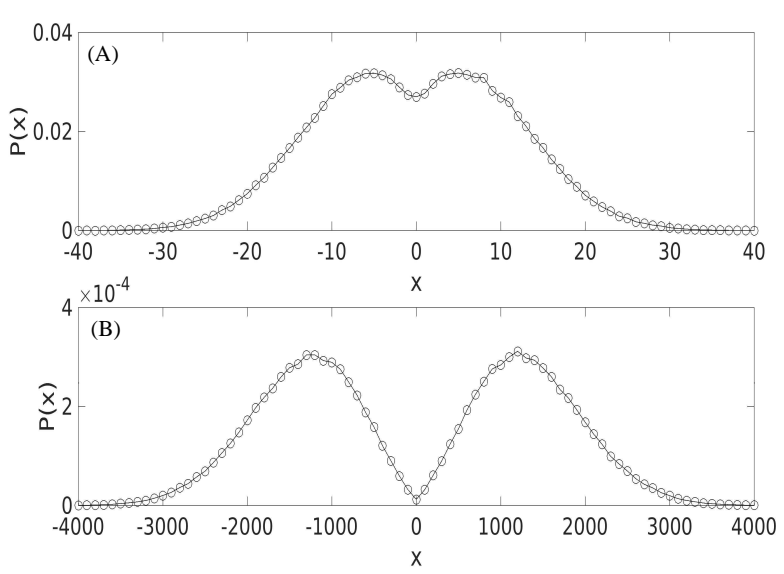

FIG. 2: The PDF at $t=10$ (A) and $t=1000$ (B) of a Brownian particle starting at the origin and moving in a medium where $\alpha(x)=1 /\left(1+x^{2}\right)$.

depict the dynamics of the particles in the system because is applies only to time-scales much larger than the ballistic time of the motion. As noted at the end of section $\llbracket$ II the ballistic distance diverges when the friction function drops faster than $x^{-1}$ at large distances. When this occurs, the velocity of the particle saturates to some finite value, and the dynamics becomes ballistic. In other words, the ballistic time diverges, and the dynamics never reaches the diffusive regime of Eq. (14).

In contrast to the diffusion equation (3), the Langevin equation (4) applies to both the ballistic and diffusive regimes. Fig 2 presents our results for the PDF of the particles at $t=10(\mathrm{~A})$ and $t=1000(\mathrm{~B})$. The results, which are based on numerical intergation of $3.25 \times 10^{5}$ trajectories starting at the origin (with velocities drawn from the standard Gaussian equilibrium distribution), demonstrate that as the time increases, the PDF becomes increasingly bimodal. This indicates the emergence of two opposite particle currents propagating away from the origin. Fig. 3(A) shows the velocity probability distribution function (VPDF) at $t=1000$ (solid circles), which is also bimodal and, thus, does not coincide with the initial equilibrium distribution (depicted by the dashed line in the figure). The VPDF at $t=2000$ (not shown) is essentially identical to the VPDF in Fig. 3(A), which proves this VPDF represents the steady state of the velocity distribution. From the steady state VPDF, we find that the steady state squared velocity $\left\langle v^{2}\right\rangle \simeq 2.41$ (in units of $\left.k_{B} T / m\right)$ and, therefore, at large times the position MSD $\left\langle x^{2}\right\rangle=\left\langle v^{2}\right\rangle t^{2}=2.41 t^{2}$. This result, which is fully corroborated by the numerical data in Fig. 3(B), demonstrates that the particles end up moving inertially with velocities drawn from the steady state VPDF.
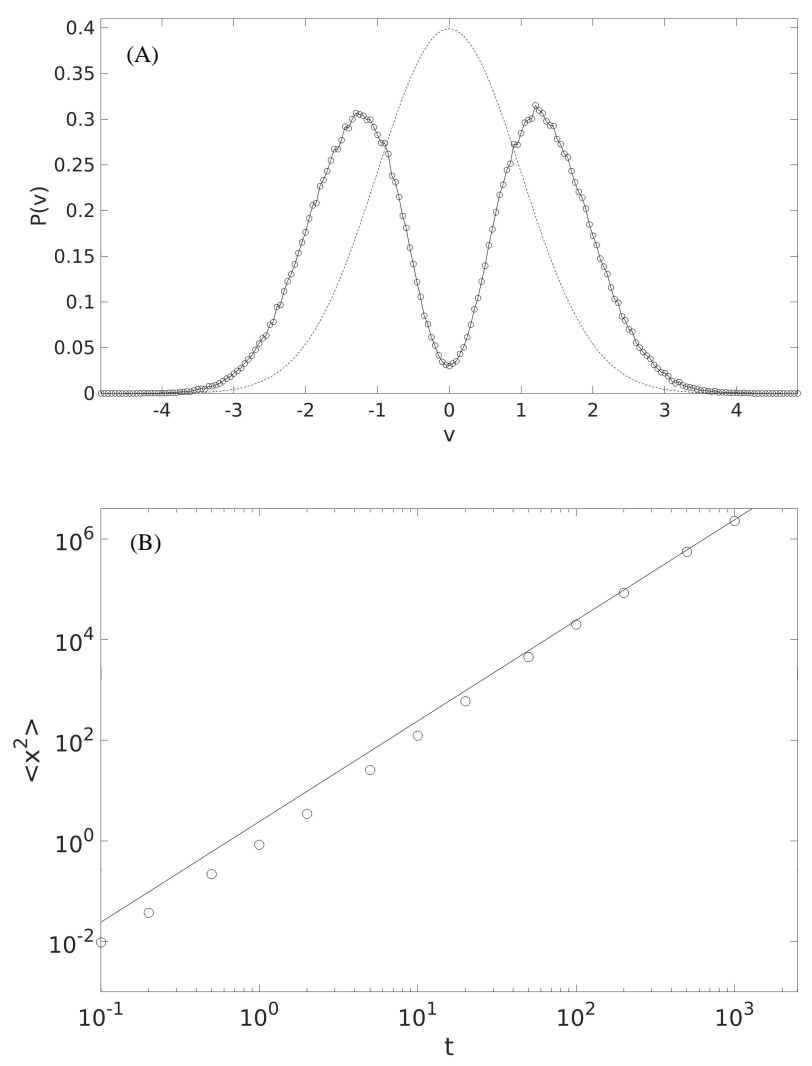

FIG. 3: (A) Circles - The bimodal VPDF at $t=1000$. The dashed line depicts the initial Gaussian equilibrium VPDF. (B) The computed MSD of the particle (circles) vs. the asymptotic power-law form $\left\langle x^{2}\right\rangle=2.41 t^{2}$ (solid line).

\section{The fluctuation-dissipation relationship}

Integrating Eq. (4) with respect to time, squaring the equation, and taking the ensemble average over noise realizations, yields the generalized form of the fluctuationdissipation relationship for systems with spatially varying friction [13], which reads

$$
\left\langle(m \Delta v)^{2}+2 m \Delta v \Delta A+(\Delta A)^{2}\right\rangle=\int_{0}^{t} 2\left\langle\alpha\left(t^{\prime}\right)\right\rangle k_{B} T d t^{\prime} .
$$

If at large times the dynamics enters the diffusive regime, the first two terms on the l.h.s. become negligible compared to the third one. Moreover, for a constant $\alpha$, the third term on the l.h.s. is equal to $\alpha^{2}\left\langle(\Delta x)^{2}\right\rangle$, while the integral on the r.h.s. gives $2 \alpha k_{B} T t$. Thus, for constant friction, expression (17) reduces (at large times) to the well-known form of the fluctuation-dissipation relation$\operatorname{ship}\left\langle(\Delta x)^{2}\right\rangle=2\left(k_{B} T / \alpha\right) t=2 D t$.

For the dynamics discussed in section IIIB the motion is not diffusive, but rather becomes ballistic at large times. However, relationship (17) holds for any time $t$, regardless of the character of the dynamics. This is nicely demonstrated in Fig. 凹(A), where we plot the ensemble 

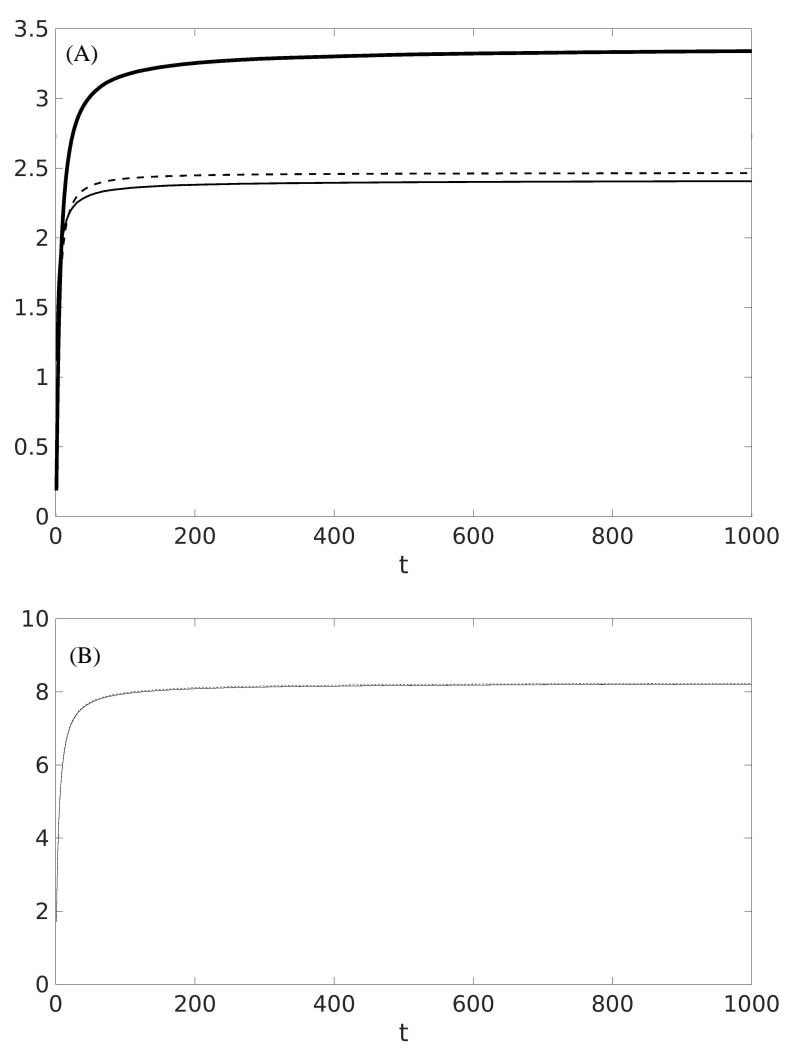

FIG. 4: (A) The ensemble averages of $(m \Delta v)^{2}$ [first term on the 1.h.s. of Eq. (17) - thin solid line], $2 m \Delta v \Delta A$ (second term - thick solid line), and $(\Delta A)^{2}$ (third term - dashed line), as a function of time $t$. (B) The sum of the three averages shown in (A) (dashed line) vs. the average of $2 \alpha k_{B} T$ integrated over time [r.h.s. of Eq. (17) - solid line], as a function of $t$.

averages of the three terms on the 1.h.s. of Eq. (17). As can be seen, all three terms grow rapidly at short times, which include the very initial ballistic segment, and the following interval of diffusive motion. At $t \gtrsim 100$, all three terms saturate, which indicates the crossover from diffusive to ballistic motion. In (B) we plot the sum of the averages of these three terms (dashed line) vs. the value of the r.h.s. of Eq. (17), which is the ensemble average of $\alpha\left(x\left(t^{\prime}\right)\right)$ integrated from the beginning of the dynamics until time $t$ (solid line). The lines overlap each other (the relative difference between them is smaller than 1\%), which demonstrates that the equality between the two sides of Eq. (17) holds at all times.

\section{SUMMARY AND DISCUSSION}

In this work we used computer simulations to study the Langevin dynamics of Brownian particles in a 1D system with a friction coefficient that varies as a powerlaw of the distance from the origin $\alpha(x) \sim|x|^{-c}$. It has been demonstrated that for $c<1$, the particle diffuses anomalously with MSD $\left\langle x^{2}\right\rangle \sim t^{2 /(2-c)}$. This result can be also derived by solving the corresponding diffusion equation (3) with diffusion coefficient $D(x)=k_{B} T / \alpha(x)$.

The diffusion equation can be formally solved for $c<2$. For $1<c<2$, the solution incorrectly predicts that the MSD grows faster than the MSD of ballistic motion. This result stems from the diffusion equation, which cannot be physically justified for time scales smaller than the ballistic time of the motion. For constant friction coefficient $\alpha$, a crossover from ballistic to diffusive motion occurs on time scales $\tau \geq m / \alpha$. In the case when $\alpha(x) \sim|x|^{-c}$ with $c>1$, the friction vanishes rapidly at large distance and an opposite crossover, from diffusive back to ballistic motion, takes place. When this happens, the diffusion equation can no longer be used if the ballistic time diverges, in which case the motion remains ballistic (as the particle escapes to infinity).

In the example discussed in section ஹIB, the divergence of the friction at the origin is removed, while at large distances $\alpha(x) \sim|x|^{-2}$. Even if the friction never vanishes completely, it drops at such a fast rate that it quickly becomes irrelevant. Thus, this example resembles dynamics of a Brownian particle in a finite fluid drop held at constant temperature $T$. When the particle reaches the surface of the drop, it escapes, and its velocity no longer changes. At the moment of escape the particle has to have a velocity component directed outwards from the drop and, therefore, the velocity distribution function outside of the drop differs from the equilibrium Gaussian equilibrium velocity distribution at temperature $T$ [see Fig. 3(A)]. Noticeably, the mean kinetic energy of the escaping particle is larger than the corresponding equilibrium value, $d k_{B} T / 2$ (where $d$ is the dimentionality of the system). The fact that, on average, the escaping particle takes away an amount of kinetic energy larger than the equilibrium value implies that the molecules of the fluid drop are left with an average kinetic energy smaller than the equilibrium value. The drop cools down slightly, and in order to maintain the temperature at $T$, it must be connected to a true heat reservoir that would supply the missing energy. This consideration is missing in the framework of Langevin's equation that completely neglects the influences of the Brownian particle on the surrounding medium.

Finally, we note that, within the framework of Langevin dynamics, a generalized form of the fluctuation-dissipation relation has been previously derived [Eq. [17)]. This form holds for dynamics in media with spatially varying friction, at all times (i.e., both within the ballistic and diffusive regimes of the dynamics). If $\alpha(x)$ is bound between two positive values, the motion at large times becomes diffusive. In such a case, the 1.h.s. of Eq. (17) becomes dominated by the third term, and both sides of the equation grow linearly with $t$. Anomalous diffusion is observed when at large distances $\alpha(x) \sim|x|^{-c}$ with $c<1$. In this case, the third term still dominates the other two terms on the l.h.s.; however, the large time behavior scales like $x^{2(1-c)} \sim t^{2(1-c) /(2-c)}$. For $c \geq 1$, the motion becomes ballistic at large times. 
In this case, all terms on the l.h.s. are equally important and, like the r.h.s. of the equation, relax to constant asymptotic values.

Verlag, Berlin, 1988).

[16] P. Hänggi, Helv. Phys. Acta 51, 183 (1978).

[17] Stochastic differential equations can be used to describe different processes in diverse fields, such as biology, economy, neuroscience, geophysics, and more. For many applications, other interpretations (e.g., Itô or Stratonovich) may be appropriate. Here, we consider the physical problem of a particle's dynamics in isothermal systems, for which the relevant interpretation is the one of Hänggi. When the particle moves in a potential energy field $U(x)$, a force term $f(x)=-d U / d x$ should be added to the r.h.s. of Eq. (4). At large times, one expects to approach the equilibrium Boltzmann distribution function $P(x) \sim \exp \left[-U(x) / k_{B} T\right]$, which does not depend on the diffusivity function $D(x)$. This is achieved with the Hänggi conventions, while other interpretations require the addition of a spurious drift term to the Langevin equation (see [11, 12] for a more detailed discussion).

[18] N. Grønbech-Jensen, and O. Farago. Mol. Phys. 111, 983 (2013).

[19] N. Grønbech-Jensen, N. R. Hayre, and O. Farago, Comput. Phys. Commun. 185, 524 (2014).

[20] A. G. Cherstvy , A. V. Chechkin, and R. Metzler, New J. Phys. 15, 083039 (2013).

1093 (2014).
[14] J. L. Domb, Ann. Math. 43, 351 (1942).

[15] H. Risken, The Focker-Planck Equation (Springer- 